\title{
Geospatial Intelligence in Dealing with COVID-19 Challenges in Czechia
}

\author{
Milan Konecny, Jiri Hladik, Jiri Bouchal, Lukas Herman and Tomas Reznik
}

This chapter deals with aspects of geospatial support to manage COVID-19 in Czechia. The first three cases of the disease were confirmed on March 1, 2020. Geospatial intelligence has played an important role in analysing and predicting the spread of the disease, in designing and optimizing measures against it, and in reducing harm. Three categories of applications were identified and described when following the conducted analysis of the content and purpose of COVID-19-related geospatial applications and solutions. The first, the visual analytics of COVID-19-related health statistics follows the concept of multiple coordinated views and dynamic queries. This category of applications uses various official or semi-official sources of geospatial data and their presentation in interactive web maps. The second, different tracking applications or tools for analysis of people's movement and identification of risky contacts was implemented. In the Czechia, these are for example eRouska mobile application and movement tracking in the Mapy.cz application. The third category represents decision support systems for public administration, emergency services and volunteers. Example of this category is the interactive map of registered volunteers used by coordination centre established by Masaryk University (MUNI) in Brno, Czechia. The advantages, limitations, and possible future directions of the mentioned applications of geospatial intelligence are discussed in the conclusions.

\subsection{Introduction}

The United Nations Global Geospatial Information Management (U.N. GGIM), which includes the core idea of creating a global geospatial integrative data ecosystem with potential applications in various situations (incl. pandemic) and on all geographical levels (incl. local and regional), is fundamentally based on the Spatial Data Infrastructure (SDI) concept. The COVID-19 pandemic has again highlighted the need of societies to deal with various kinds of data and to enrich and integrate them "on-line" or "near-online". One of the necessary preconditions is to analyse data fast and provide visual representations such as digital maps and other types of models. Geospatial intelligence proved itself to be an useful bridge combining the already existing authoritative spatial data (as in the Infrastructure for Spatial Information in the European Community - INSPIRE and national SDIs) with referenced health information regularly collected by special governmental institutions (in Czechia, this is e.g. the Institute for Health Information and Statistics - IHIS) or obtained by everyday measurements based on COVID-19 testing among various population groups and regions of Czechia. This paper describes theoretical and practical approaches based on state-of-the-art geospatial intelligence. 
Based on the analysis of the content and purpose of existing and newly emerging geospatial applications and solutions [1-3], the authors of this paper have integrated three approaches to improve dealing with COVID-19-related problems:

- Visual analytics of COVID-19-related health statistics,

- Tracking and analysis of people's movement and identification of risky contacts, and

- Decision support systems for public administration, emergency services and volunteers.

\subsection{Visual Analytics of COVID-19-related Health Statistics in Czechia}

The main goal of visual analytics is to provide interactivity through utilizing the concept of multiple coordinated views and dynamic queries to emphasize the impact of changes in analysed phenomena. A number of applications have been developed around the world for the visual analysis of geospatial data on the spread of COVID-19 [1-3]. Many of these applications are global and use data from the World Health Organization (WHO). Several applications use data from Johns Hopkins University in Maryland; this data source is created by combining several primary data sets (such as WHO) by supplementing them with additional information. In Czechia, applications that visualise global data have also been developed alongside visualisations built to analyse domestic data. Those focused on Czechia primarily use the data provided by the Ministry of Health of the Czech Republic, as well as the IHIS data, which are included. Data collection process includes an aggregation of data from individual Regional Public Health Stations. These data are freely available [3].

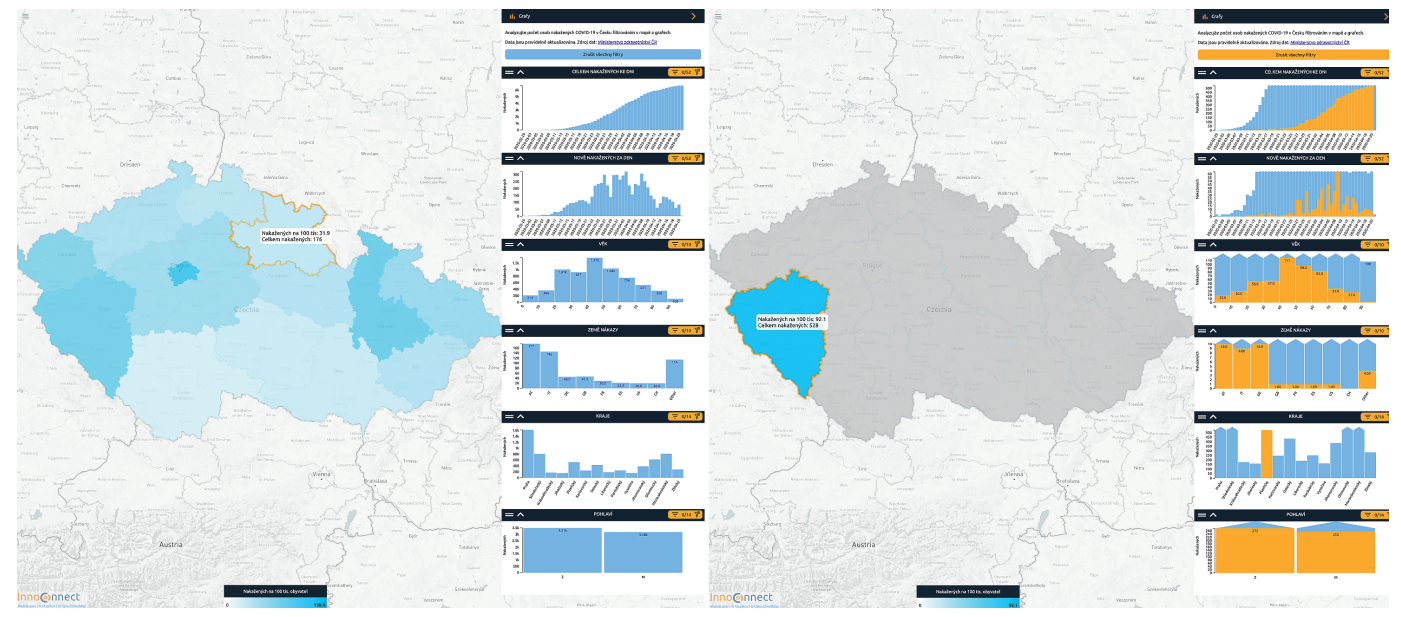

\section{FIGURE 30.1}

An interactive map of COVID-19 in Czechia ${ }^{*}$. Note: choropleth maps show the number of infected per 100 thousand inhabitants. Graphs in columns are used to select according to the infected persons' age, region and gender, or according to the date and source country (if infected abroad) of the infection. The map on the left shows the overall situation in Czechia; the situation in the Pilsen Region is displayed on the right.

*http: //mapa-koronavirus. innoconnect. net

A map of COVID-19 spread in Czechia $^{1}$ is an example of this kind of visual analytics application.

\footnotetext{
${ }^{1}$ https://mapa-koronavirus.innoconnect.net/
} 
The map allows to analyse the number of people infected by COVID-19 through implementing multiple linked views to present the data. Each of the views (map and charts) enables different interactions, such as brushing, relationship analysis, and filtering that trigger an instant update of the other views. Different combinations of filters can be applied for deeper insights, for instance by selecting a single source country of infection: the distribution of cases originating in that country is then visualised according to time, region, gender and age (Figure 30.1). It is also possible to select people over 70 years of age as a group and highlight the regions with the highest number of infected senior citizens. These functionalities are implemented on top of the WebGLayer open source library ${ }^{2}$.

\subsection{Tracking and Analysis of People's Movement and Determination of Risky Contacts}

The application and procedures described in this section form the technological basis for the deployment of the "smart quarantine system". The main part of this system in Czechia consists in creating "memory maps" based on processing of an individual's movement data obtained from the relevant mobile operator. There are two facts that need to be emphasized. The individual must agree to data processing and no data belonging to any other persons are used in the process. The aim is, therefore, to help the person remember all the places he or she visited and then identify all people (s)he came into contact with. The use of memory maps is supplemented by other voluntary technologies such as the eRouska (eMask in English) mobile application ${ }^{3}$ and movement tracking in the Mapy.cz application ${ }^{4}$.

eRouska is a mobile application for smartphones that helps authorized officers from Regional Public Health Stations to easily and quickly identify people with whom an infected person came into contact with and who are therefore also potentially at risk of infection. The application uses Bluetooth and records close contact with other users of the application. Tracking within Mapy.cz uses location history data collected by mobile devices. Thanks to location sharing, an increased probability of infection can be calculated; this probability is determined on the basis of whether the observed person stayed in the same place for a significant period of time as a person who has tested positive for COVID-19. By July 2020, 1.4 million users have volunteered to share their location data using the Mapy.cz application (out of the total of 10.7 million inhabitants of Czechia).

The aforementioned tracking data from mobile phones can also be used to improve the population estimates in certain areas, e.g. due to the increased incidence of COVID-19 in these areas. Kubicek et al. [4] describe a proof-of-concept application of tracking data from mobile phones in a crisis management context. A similar approach can also be used in the future to improve the estimates of current population in smaller areas affected by COVID-19 in order to facilitate closure planning or to plan services.

\subsection{Decision Support Systems for Public Administration, Emergency Services and Volunteers}

Geospatial applications can also serve as a platform for volunteers, community groups and those who need help in connecting with each other, as well as for local authorities and individuals or groups of volunteers matching specific locations and other criteria. Maps can display information and help in navigation to hospitals, clinics, grocery stores or pharmacies, places where personal protective

\footnotetext{
${ }^{2}$ http: //webglayer.org/

${ }^{3}$ https://erouska.cz/en

${ }^{4}$ https://napoveda.seznam.cz/en/mapy/covid-19/
} 
equipment can be purchased and similar. In affected areas, this information and connections could improve the organization of assistive services and thus potentially save lives.

Masaryk University (MUNI) in Brno, Czechia, has established a coordination center for volunteers right at the beginning of the COVID-19 crisis $^{5}$. The help offered consisted mostly in the manufacture of face masks, food or medicine delivery to vulnerable people, babysitting, teaching assistance and similar. Both volunteers and requests for help and assistance were collected through online forms, e-mails and phone calls. All data thus obtained included geospatial information; Geographic Information System (GIS) support has been introduced across all these activities to increase the coordination center's efficiency.

An interactive web map based on the Leaflet library ${ }^{6}$ has been established to display the location of all registered volunteers. The map also features browsing by attributes and filtering and address search to help operators locate people who are asking for help and to assign a suitable volunteer nearby (Figure 30.2). Input data from Excel sheets were processed using a Python script. To obtain geographic information from the addresses provided, two geocoding services are used: the national Registry of Territorial Identification, Addresses and Real Estate - RUIAN geocoding service $^{7}$ operated by the Czech Office for Surveying, Mapping and Cadastre on the one hand, and the geocoding service provided by the Czech online and mobile map application Mapy.cz ${ }^{8}$ developed by the private company Seznam.cz on the other hand. The output point dataset was visualized using Leaflet.markercluster plugin ${ }^{9}$. The data processing script was executed automatically every 10 minutes to keep the data in the application up-to-date.

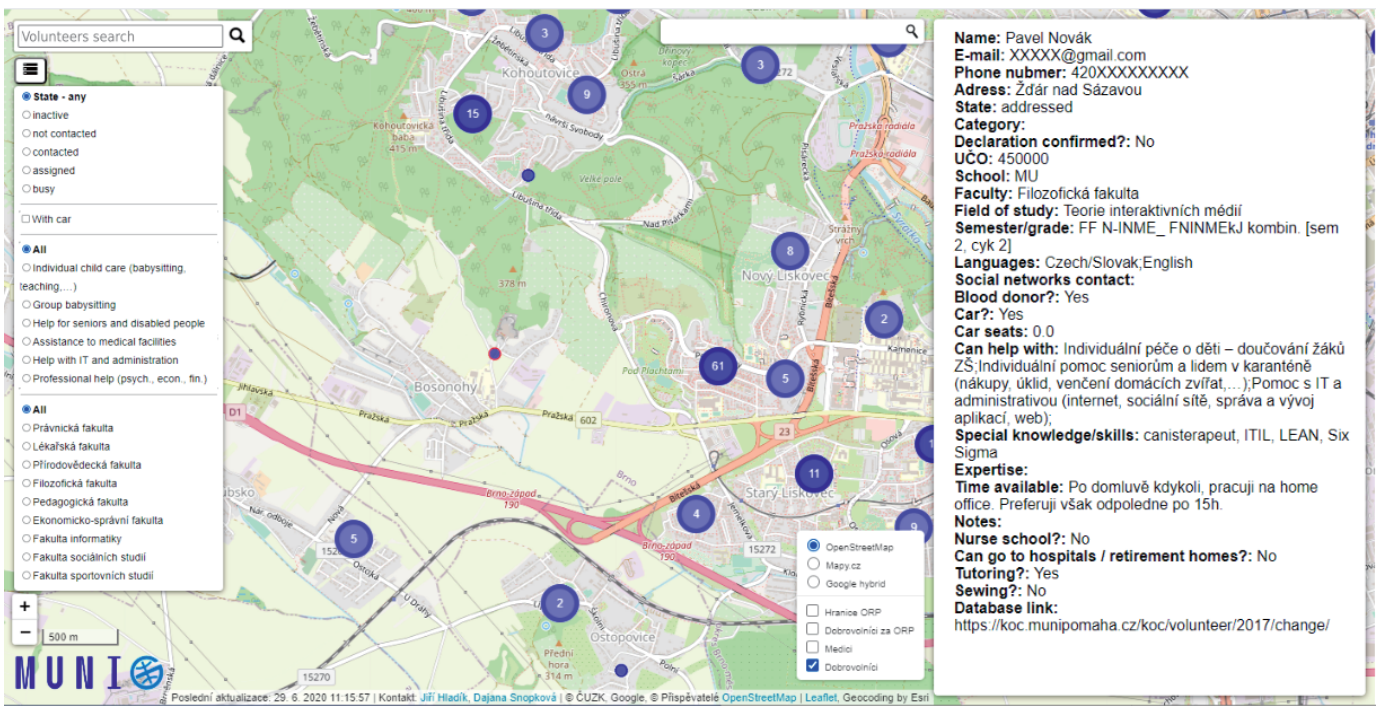

FIGURE 30.2

An interactive map of registered volunteers. Note: elements on the left contain a search bar and data filtering; the right panel contains information on the selected volunteer.

A similar application was established for public use in order to promote the activities of the coordination center and, simultaneously, to invite more help-seekers by providing information on available volunteers nearby. To ensure protection of the volunteers' privacy, the amount of sensitive information was reduced, location data were anonymized and aggregated. The internal version of the map of volunteers was used by 30 people, usually 7 workers per day. Overall, more than 4,000 volunteers have registered, and the center has successfully resolved 2,405 requests for help. The

\footnotetext{
${ }^{5}$ https://munipomaha.cz/en/i-need-help

${ }^{6}$ https://leafletjs.com/

${ }^{7}$ http://ags.cuzk.cz/arcgis/rest/services/RUIAN/Vyhledavaci_sluzba_nad_daty_RUIAN/MapServer

${ }^{8}$ https://api.mapy.cz/geocode

${ }^{9}$ https://github.com/Leaflet/Leaflet.markercluster
} 
map has also helped coordinate efforts with other volunteer groups where they had a shortage of people.

\subsection{Conclusions and Discussion}

Geospatial information has been an irreplaceable tool across the activities to combat the COVID-19 pandemic in Czechia, regardless of whether the particular application has been provided centrally by the government/public administration, the private sector and/or volunteer activities. Location information, mapping and GIS tools have been used at all stages, from preparedness in areas without infected persons, through response at hotspot areas, to mitigation across the whole country. Open source as well as customized national commercial products have been used with similar frequency. For such purposes, both, national map data sources/registries and open data like OpenStreetMap are combined.

Several applications have been created quickly and spontaneously, many of them initially containing several cartographic mistakes or shortcomings that were later gradually corrected. A typical shortcoming was, for example, the use of a choropleth map to display the absolute number of infected people instead of showing a share of the infected in the population, or using proportional map symbols. Geospatial applications visualizing COVID-19-related health statistics have also suffered from data quality issues such as different data reporting practices in different regions. Potentially, data errors can also occur in processing or in combining input data from different sources.

This use of Geospatial Intelligence based on a combination of the three approaches in dealing with the COVID-19 pandemic represents one of many possibilities. It can also be applied in other crisis situations, other cities, countries and continents if high-quality geospatial data are available.

The tasks related to the COVID-19 pandemic were in public administration and public health bodies firstly addressed via spreadsheets, notes etc. Geospatial intelligence was employed in early stages of the pandemic as it resulted in such bodies in (1) significantly faster processing, (2) more complex tasks, (3) sophisticated predictions and (4) shortening the supply chain. All these benefits are demonstrated during the second wave of the COVID-19 pandemic ${ }^{10}$ that is even stronger than the first wave in spring 2020 .

\section{Acknowledgement}

This research was supported from the European Union's Horizon 2020 research and innovation programme under grant agreement No 769608 titled Policy Development based on Advanced Geospatial Data Analytics and Visualisation (PoliVisu).

\section{References}

[1] Maged N. Kamel Boulos and Estella M. Geraghty. Geographical tracking and mapping of coronavirus disease covid-19/severe acute respiratory syndrome coronavirus 2 (sars-cov-2) epidemic and associated events around the world: how 21st century gis technologies are supporting the global fight against outbreaks and epidemics. International Journal of Health Geographics, 19(1):8, Mar 2020. ISSN 1476-072X. doi: 10.1186/s12942-02000202-8.

\footnotetext{
${ }^{10}$ As witnessed in the Czechia from the end of August 2020.
} 
[2] Amy L. Griffin. Trustworthy maps. Journal of Spatial Information Science, 2020(20):5-19, 2020. ISSN 1948-660X. doi: 10.5311/JOSIS.2020.20.654.

[3] Vit Pászto, Jaroslav Burian, and Karel Macku. Covid-19 data sources: Evaluation of map applications and analysis of behaviour changes in europe's population. Geografie (Utrecht), 125(2):171-209, 2020. doi: https: //doi.org/10.37040/geografie2020125020171.

[4] Petr Kubíček, Milan Konečný, Zdeněk Stachoň, Jie Shen, Lukáš Herman, Tomáš Řezník, Karel Staněk, Radim Stampach, and Šimon Leitgeb. Population distribution modelling at fine spatio-temporal scale based on mobile phone data. International Journal of Digital Earth, 12(11):1319-1340, 2019. doi: 10.1080/17538947.2018. 1548654 . 